\title{
Exogenous lipoid pneumonia associated with oil pulling: Report of two cases
}

\author{
Chi Fong Wong1, See Wan Yan1, Wai Mui Wong1, Ronnie S.L. Ho2 \\ 1 TB and Chest Unit, Grantham Hospital, Aberdeen, Hong Kong \\ 2 Department of Pathology, Queen Mary Hospital, Pokfulam, Hong Kong
}

\begin{abstract}
Two ladies with history of carcinoma of tongue presenting with unresolving pneumonia were ultimately diagnosed to have lipoid pneumonia, and both were subsequently found to be associated with the practice of oil pulling which is a popular complementary therapy. Apart from cessation of oil pulling, they were treated with repeated therapeutic lobar broncho-alveolar lavage. Despite the potential benefits of oil pulling on oral health, people especially those at risk of aspiration, should be properly informed of this potential risk when considering this form of complementary therapy.
\end{abstract}

\section{Introduction}

Lipoid pneumonia is an uncommon non-infectious pneumonia characterized by the presence of lipid-laden macrophages or free lipid in the alveoli. Exogenous lipoid pneumonia refers to the situation when the lipid is from an exogenous source. We herein report two cases of exogenous lipoid pneumonia associated with the practice of oil pulling.

Corresponding author: Dr. Wong Chi Fong, TB and Chest Unit, Grantham Hospital, 125 Wong Chuk Hang Road, Hong Kong.

Fax: +852.25802493. E-mail: wongcf2001@yahoo.com.hk

Key words: Lipoid pneumonia; oil pulling.

Contributions: CFW, SWY, physicians attending the cases, involved in diagnosis and management; CFW, SWY, WMW, manuscript drafting; RSLH, pathologist involved in management, provision of the pathological slides, contribution to manuscript drafting.

Received for publication: 2 March 2018

Accepted for publication: 2 August 2018

CCopyright C.F. Wong et al., 2018

Tipografia PI-ME Editrice, Italy

Monaldi Archives for Chest Disease 2018; 88:922

doi: 10.4081/monaldi.2018.922

This article is distributed under the terms of the Creative Commons Attribution Noncommercial License (by-nc 4.0) which permits any noncommercial use, distribution, and reproduction in any medium, provided the original author(s) and source are credited.

\section{Case \#1}

A 50-year-old lady, who had history of carcinoma of tongue treated with partial glossectomy and neck dissection followed by adjuvant chemo-radiotherapy 10 years ago, presented with right lower lobe pneumonia 2 years ago. Swallowing assessment showed severe oro-pharyngeal dysphagia with high risk of aspiration. She was therefore referred and had a percutaneous gastrostomy done and with feeding all through gastrostomy since then. However, she continued to experience repeated episodes of pneumonia affecting right middle and lower lobes. It was initially thought to be related to silent aspiration of regurgitated milk after gastrostomy feeding. Despite adjustment of the scheduled feeding time and volume, and advice on the proper posture during and after feeding, the symptoms did not improve and radiological features of right middle and lower lobe consolidation persisted (Figure 1). Fiber-optic bronchoscopy showed swollen mucosa of right lower lobe bronchus and biopsy revealed the presence of foreign body reaction and granulomatous inflammation. Based on the biopsy findings, non-infective inflammatory lung condition was suspected and she was tried on systemic steroid treatment. However, there was no improvement after 3 months of steroid trial.

On further detailed questioning, she admitted having the habit of practicing regular oil pulling at home for over 2 years. Bronchoscopy was repeated and the broncho-alveolar lavage (BAL) fluid showed the presence of fat substance on gross examination (Figures 2 and 3). On cytological examination with Oil Red stain, abundant fat globules were found (Figure 4). The diagnosis of exogenous lipoid pneumonia associated with oil pulling was made.

\section{Case \#2}

Another middle-aged lady with past history of thyrotoxicosis, atrial fibrillation, old pulmonary tuberculosis and carcinoma of tongue two years ago with hemi-glossectomy, presented similarly with cough. Chest x-ray and CT thorax showed the presence of right middle lobe consolidation with enlarged mediastinal lymphadenopathy. The consolidation did not resolve with antibiotic therapy.

Repeated investigations were non-revealing and she ultimately underwent a surgical lung biopsy which revealed granulomatous inflammation on histology with tissue culture growing Mycobacterium abscessus. She received anti-mycobacterial treatment for more than 12 months. However, there was persistent consolidation at right middle lobe on radiographs (Figure 5) despite repeated sputum cultures were negative for mycobacteria. Swallowing assessment showed only minimal oro-pharyngeal dysphagia.

On direct questioning, she also admitted having been practicing regular oil pulling at home for more than a year. Bronchoscopy was performed and the BAL fluid showed abundant fat globules compatible with the diagnosis of exogenous lipoid pneumonia associated with oil pulling. 


\section{Subsequent management}

Both patients were advised to stop the practice of oil pulling. As case \#1 had received a course of steroid with no improvement, she was treated with therapeutic lobar broncho-alveolar lavage. The procedure

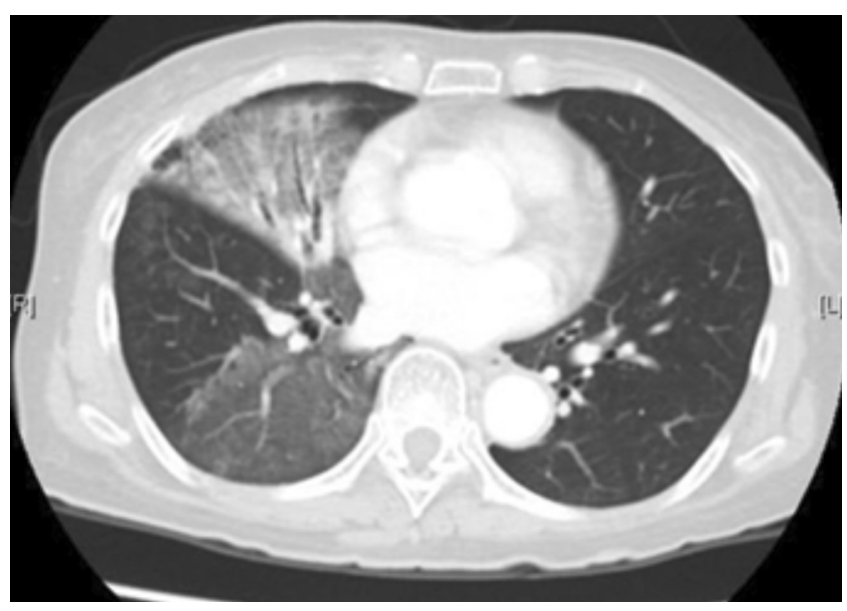

Figure 1. CT thorax of case \#1 at presentation.

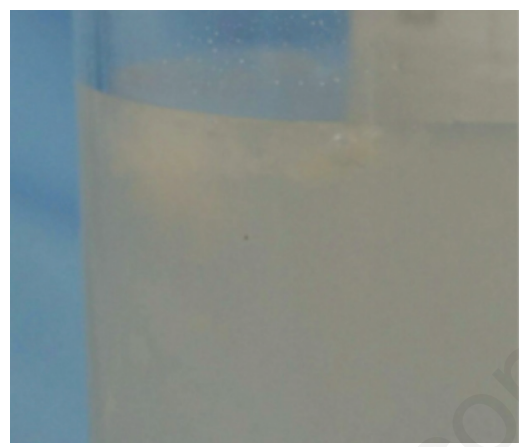

Figure 2. Gross appearance of the broncho-alveolar lavage fluid on return.

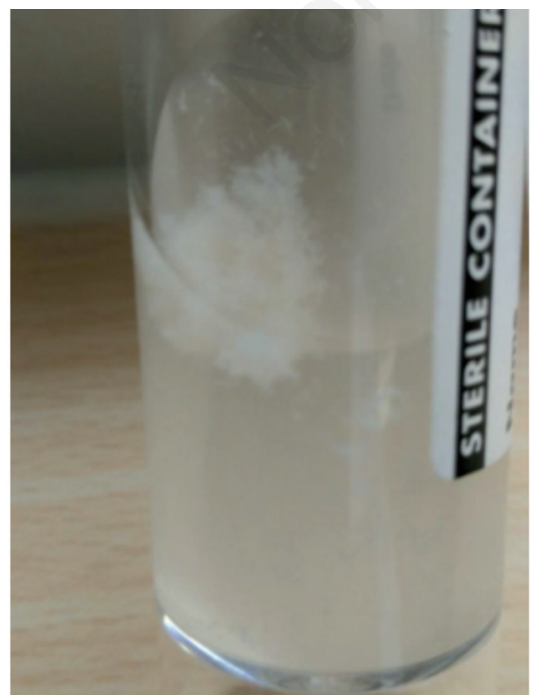

Figure 3. Gross appearance of broncho-alveolar lavage fluid on standing overnight. was performed in bronchoscopy suite under conscious sedation and local anaesthesia. Patient was given supplementary oxygen and fibreoptic bronchoscope was inserted into the airway. The tip of the bronchoscope was wedged at the orifice of the affected bronchopulmonary segments. Saline warmed to body temperature in $20 \mathrm{ml}$ aliquots was repeatedly instilled and gently sucked back. Seen under the bronchoscope, the returned fluid was mildly turbid in appearance and whitish flakes of fat-like material were seen coming out from within the affected airways. The procedure was stopped after about three to four hundred $\mathrm{ml}$ of saline had been instilled and when the returned fluid appeared clear with no more flakes of fat-like material seen. On standing, fat-like substance was seen floating on the surface of the returned BAL fluid. The procedure was very well tolerated and was repeated at 2 to 3 weekly intervals for total 5 times. For case \#2, she was treated with the same procedure of therapeutic lobar BAL. She also tolerated the procedure very well and it was repeated for total 4 times. Clinically, both patients showed improvement in symptoms of cough and shortness of breath after stopping oil pulling practice and the treatment of therapeutic lobar BAL. Chest X-ray showed marked resolution of the consolidative changes (Figures 6 and 7). Both patients remained well on follow up for over a year.

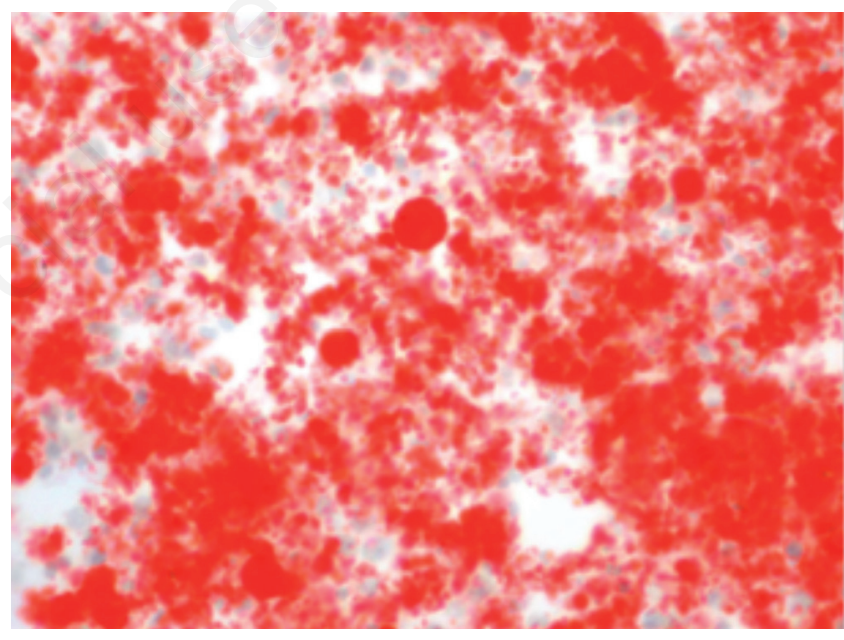

Figure 4. Oil Red stain of broncho-alveolar lavage fluid.

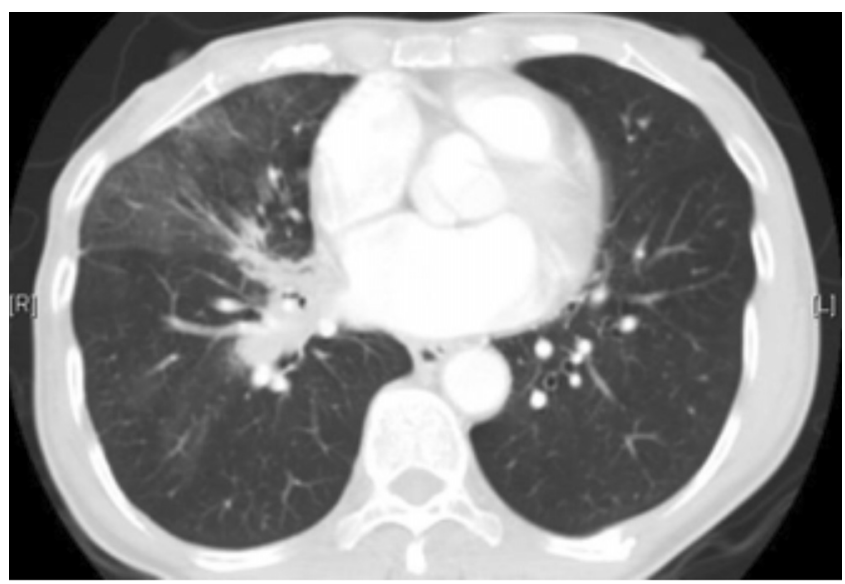

Figure 5. CT of case \#2 at presentation showing consolidation at right middle lobe. 


\section{Discussion}

Lipoid pneumonia is an uncommon disease characterized by the presence of lipids in the alveoli either in the free form or in the form of lipid-laden macrophages. It can be divided into endogenous or exogenous in aetiologies. While endogenous lipid pneumonia is usually sec- ondary to endobronchial obstructions with the lipid within alveoli arising from degenerated cells including surfactant from Type II pneumocytes, it could also be found in some chronic infectious conditions, in association with alveolar proteinosis and in some lipid-storage diseases [1]. Exogenous lipoid pneumonia is caused by exogenous lipidcontaining substance being either inhaled or aspirated into the airways.

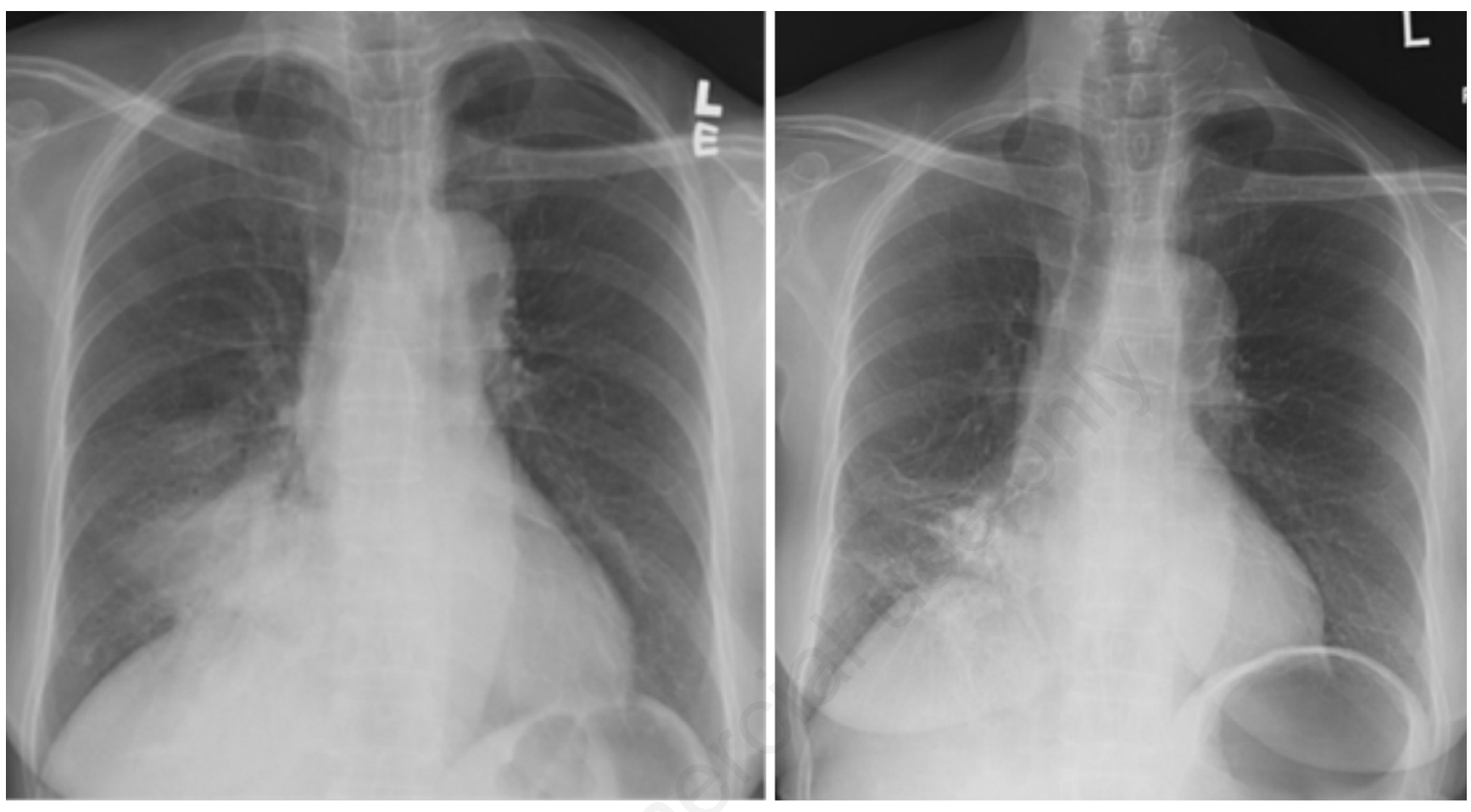

Figure 6. Chest X-ray of case \#1 at presentation and after cessation of oil pulling and therapeutic broncho-alveolar lavage.
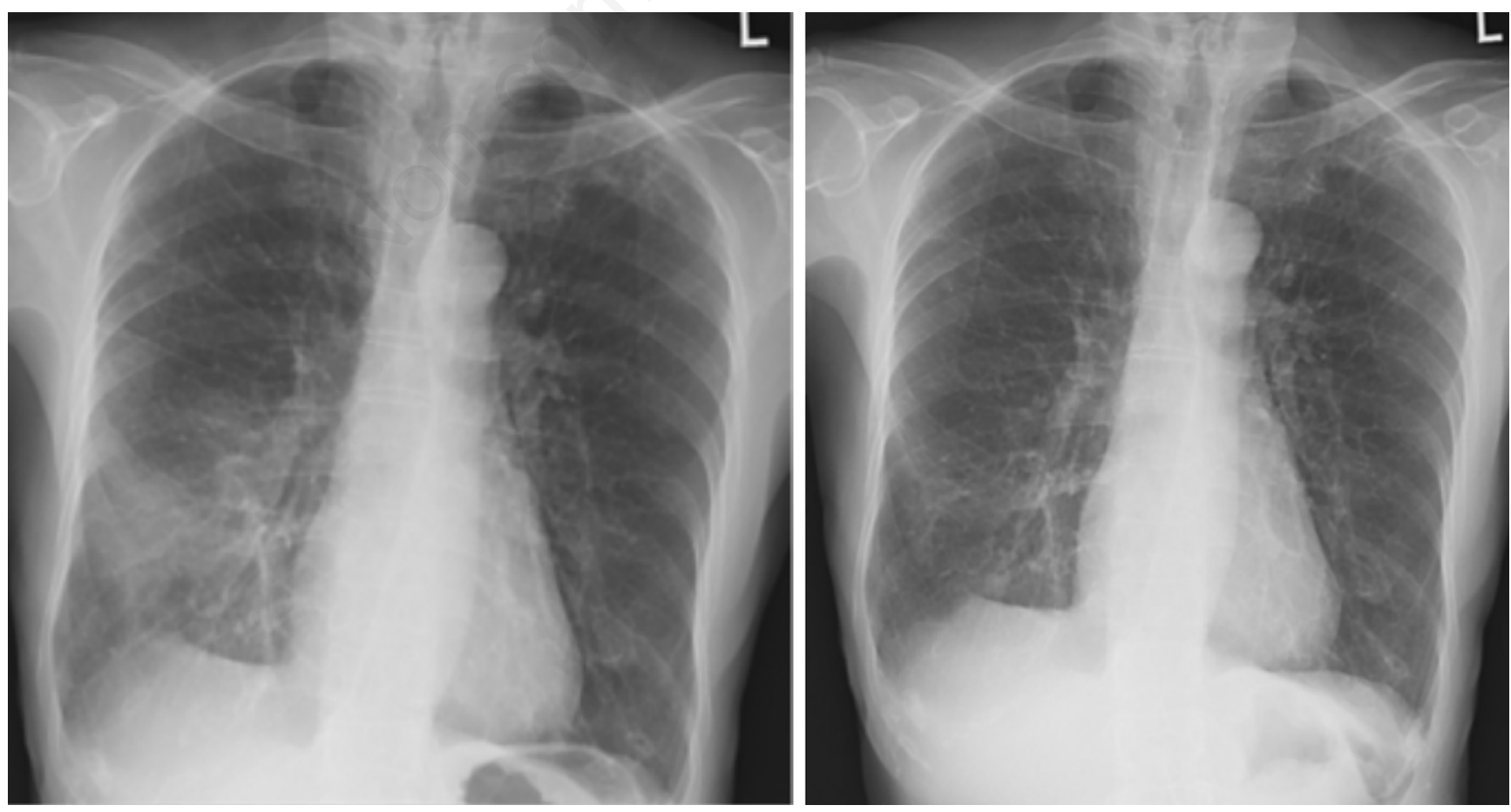

Figure 7. Chest $x$-ray of case \#2 at presentation and after cessation of oil pulling and therapeutic broncho-alveolar lavage. 
The diagnosis of exogenous lipoid pneumonia is challenging. It usually requires the history of exposure to lipid substance, the presence of compatible radiological appearance and the demonstration of the presence of lipid in the alveoli [2]. The history of oil exposure could be very trivial and is not easily revealed. The histological diagnosis might be missed if the specimen is processed in the usual paraffin blocks as the lipid can dissolve in the paraffin. Special oil stains on fresh lung biopsy or BAL fluid can help to demonstrate the presence of lipids.

On the aetiologies of exogenous lipoid pneumonia, it has been reported to be caused by various types of lipids and oils, including the use of liquid paraffin as laxatives, oil-based nose-drops, or inhalation of insecticide or kerosene [1].

Oil pulling is a traditional folk remedy practiced in ancient India and has been described in ancient Ayurvedic text. The procedure involves putting a tablespoon of oil (coconut, sesame or sunflower oil) into and swished around the mouth for about $20 \mathrm{~min}$ in the morning. The oil is spitted out afterwards and mouth rinsed with warm saline or water. It is claimed that through this procedure, toxins in the body are being "pulled" into the oil and thus one's health restored. Published scientific studies have shown that this practice can improve the oral health by reducing the bacterial count within oral cavity and plague formation [3]. For fear of the side effects of conventional modern medicine, people nowadays have been showing more interest in traditional practices as complementary forms of therapy for promoting one's health. Oil pulling, by its simplicity in practice and the oil involved being natural products, has been widely popularized in the media and internet in the recent years. However, this practice is not without risks as there have been case reports of lipoid pneumonia associated with oil pulling [4,5].

The two cases reported here shared several features in common: both are middle-aged, educated and elegant ladies. Also, both had recovered from cancer of tongue. The changes in the oral cavity after tongue cancer treatment might prompt them to try measures to improve their oral health. However, the structural changes in the mouth and the associated swallowing dysfunction might put them at risk of aspiration despite the oil was supposedly spitted out and cleared from the mouth each time.

\section{Conclusions}

We have reported two cases of lipoid pneumonia associated with the practice of oil pulling in patients with history of carcinoma of tongue. Despite the potential benefit of oil pulling on oral health, people especially those at risk of aspiration, should be properly informed of this potential risk when considering this form of complementary therapy.

\section{References}

1. Hadda V, Khilnani GC. Lipoid pneumonia: an overview. Expert Rev Resp Med 2010;4:799-807.

2. Marchiori E, Zanetti G, Mano CM, Hochhegger B. Exogenous lipoid pneumonia. Clinical and radiological manifestations. Respir Med 2011;105:659-66.

3. Vagish Kumar L. Shanbhag. Oil pulling for maintaining oral hygiene - A review. J Tradit Complement Med 2017;7:106-9.

4. Kim JY, et al. Recurrent lipoid pneumonia associated with oil pulling. Int J Tuberc Lung Dis 2014;18:251-2.

5. Kuroyama M, et al. Exogenous lipoid pneumonia caused by repeated sesame oil pulling: a report of two cases. BMC Pulm Med 2015;15:135. 